\title{
Searching for Extreme SEP Events with STEREO
}

\author{
C.M.S. Cohen ${ }^{1 a}$, J.G. Luhmann ${ }^{b}$, R.A. Mewaldt ${ }^{a}$, M.L. Mays ${ }^{c}$, H.M. Bain ${ }^{d, e}$, Y. Li ${ }^{b}$, \\ C.O. Lee L $^{\text {b }}$ \\ ${ }^{a}$ California Institute of Technology, Pasadena, CA, USA \\ ${ }^{b}$ University of California Berkeley, Berkeley, CA, USA \\ ${ }^{c}$ GSFC/Catholic University, Laurel, MD, USA \\ ${ }^{d}$ University of Colorado, Boulder, CO, USA \\ ${ }^{e} N O A A / S W P C$, Boulder, CO, USA \\ E-mail: cohenesrl.caltech.edu
}

Ground Level Enhancement (GLE) events are a particular class of solar energetic particle (SEP) events in which the particle spectrum is hard enough to produce effects on the Earth's surface. Historically a GLE had to be observed by two separate ground-based monitors to be added to the informal 'GLE list' (http://neutronm.bartol.udel.edu/ pyle/GLE_List.txt). As these events are among the most energetic of SEP events, they are a clear space weather concern and understanding the conditions under which they occur and the processes that create them is critical for improving our predictive capabilities. By their very definition, GLEs cannot be identified with only space-based instrumentation or at locations far from Earth, yet as we increasingly send instrumentation to distant locations, our need for predicting space weather throughout the heliosphere becomes more urgent.

Using the 13-100 MeV proton intensities observed during the 13 Dec 2006 GLE by the STEREO-B spacecraft (when it was still close to Earth) as a guide, we have identified several SEP events observed by STEREO of similar or larger intensity. None of these events were strong enough at Earth to register as a GLE, but had Earth been in the STEREO location, they might have been. We have calculated the eventintegrated proton spectra for these events and found they fell into two categories: those with hard spectra and those with soft spectra above a few tens of MeV. Extrapolation of the hard spectra to higher energies indicates that these events had $>500 \mathrm{MeV}$ fluences that exceeded those of the 13 Dec 2006 and 17 May 2012 GLE events by factors of $\sim 4$ to 500, suggesting that they would have caused GLEs had the Earth been suitably positioned. The spectral indices of these events are consistent with those found in previous studies of GLE events. Simulations using the WSA-ENLIL+Cone model indicate that the observing spacecraft was magnetically connected to both the event coronal mass ejection (CME) and a preceding $\mathrm{CME}$ during the hard spectrum events. This may suggest a favorable configuration for the generation of extreme SEP events and/or GLEs. 


\section{Introduction}

Ground-based measurements of solar energetic particles (SEPs) have existed since the 1940's, however a sufficient number of SEPs at energies greater than $\sim 500 \mathrm{MeV}$ are required to create these ground level enhancements (GLE) events (e.g., [1]) at high-latitude stations. As the spectrum of SEPs usually falls off rapidly above $\sim 100 \mathrm{MeV}$, GLE events are a sample of the more extreme SEP events and pose a considerable space weather concern. With their hard spectra and high intensities at energies above a few hundred $\mathrm{MeV}$, they can inflict significant damage on space-based instrumentation, astronauts and even passengers and crew on polarroute airline flights. Understanding the generation of these events is critical to their prediction as well as advancing the general knowledge of particle acceleration. Unfortunately, studying GLE events is hampered by the fact that they are relatively rare. Solar cycle 23 had 16 GLEs, while the relatively weak cycle 24 has had only 2 , resulting in 71 in the last $\sim 75$ years [2].

Several characteristics (as measured in-situ) of GLE events distinguish them from other large SEP events. A survey by [3] examined the spectra and composition of 16 GLE events and compared them to a sample of large non-GLE SEP events. They found that the GLE event spectra were best fit by a double power-law form [4] and the spectra above the break energy were signifcantly harder than those of non-GLE events. Compositionally, on average the $\mathrm{Fe} / \mathrm{O}$ and $\mathrm{Ne} / \mathrm{O}$ abundance ratios were higher in GLE events as were the deduced Fe mean charge states. The study of [5] found the solar sources of GLE events often tend to be active regions in the western hemisphere of the Sun where there is generally a direct magnetic connection between the Earth and the Sun.

Although technically GLE events are identified through the associated counting rate increases of ground-based neutron and/or muon monitors, the Sun does not preferentially send extreme SEP events towards Earth. Thus, while they cannot be labeled as GLE events, undoubtedly there are SEP events occurring elsewhere at $1 \mathrm{AU}$ which would have been GLE events had the Earth been suitably positioned. An example of this is the extreme 23 July 2012 SEP event observed by STEREO-A [6,7]. Examining such events can contribute to the knowledgebase regarding the fast and efficient acceleration of high-energy ions and futher our capability for predicting the associated radiation hazards throughout the heliosphere.

\section{Selected Events}

Near Earth, SEP sensors on GOES and SAMPEX (while it was still operational) are capable of measuring energetic protons up to several hundred $\mathrm{MeV}$. Proton spectra from 0.1 to $\sim 400 \mathrm{MeV}$ were obtained for the last two GLE events, 13 Dec 2006 and 17 May 2012, by combining measurements from a number of near-Earth SEP instruments on ACE, GOES, and SAMPEX. Unfortunately, the SEP instrumentation on the twin STEREO spacecraft (STA and STB) is limited to proton measurements $\leq 100 \mathrm{MeV}$, making it difficult to identify SEP events with the potential to create a GLE. However, the 13 Dec 2006 event was also detected by STB when the spacecraft was still near Earth. The measured event-integrated fluences from the Low Energy Telescope (LET) and the High Energy Telescope (HET) are compared to those obtained from ACE, GOES, and SAMPEX in Figure 1 and show excellent agreement.

For this study we have used LET and HET data to examine proton spectra from $<1 \mathrm{MeV}$ to $\sim 100 \mathrm{MeV}$ and heavy ion spectra from $<1 \mathrm{MeV} /$ nuc to $\sim 30 \mathrm{MeV} /$ nuc for SEP events observed 


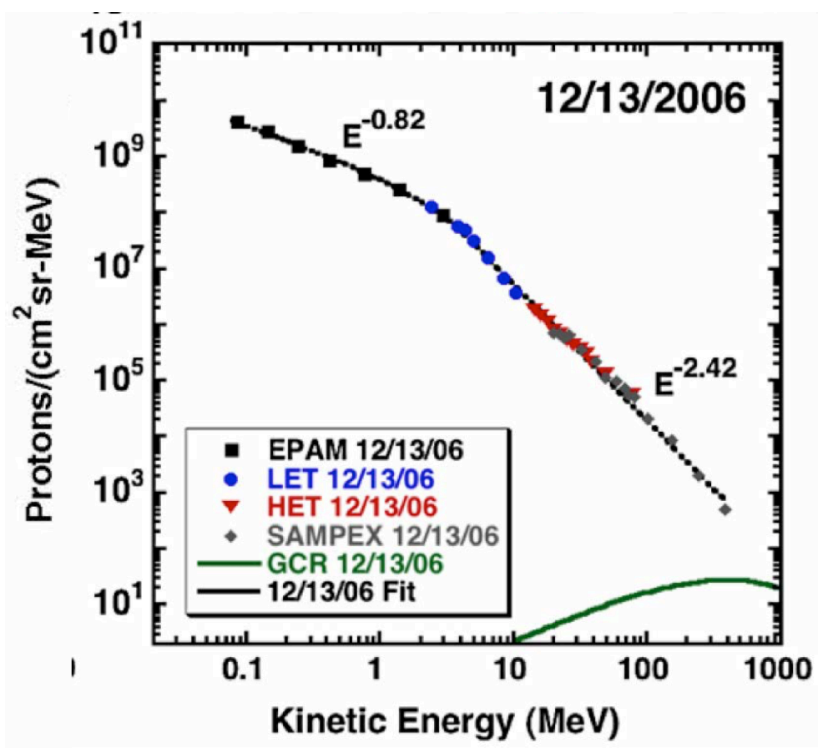

Figure 1. Event-integrated proton spectrum for the 13 Dec 2006 GLE event. The green curve also shows the GCR spectrum for reference. From [3]. by STA and/or STB. Using the STB/HET measurements during the 13 Dec 2006 event as a guide we identified events with daily intensities of $>10 \mathrm{MeV}$ protons similar to or greater than those observed during the 13 Dec 2006 event.

From this search, we have selected five events in the STEREO data that have extrapolated proton fluences at $>100 \mathrm{MeV}$ which might have been sufficient to generate a GLE had the earth been located where STEREO was. Although some of these events were also detected (with lower intensities) by near-Earth spacecraft, none of them

were identified as GLE events. Table 1 gives information for each event, specifically the date, solar source location, speed of the associated coronal mass ejection (CME), and the location of the observing STEREO spacecraft (in longitude relative to the Sun-Earth line).

Table 1: Selected Events

\begin{tabular}{|l|c|c|c|}
\hline Event Date & Source Longitude & CME velocity (km/s) & Spacecraft Longitude \\
\hline 4 Jun 2011 & W165 & 2425 & STA W95 \\
\hline 7 Mar 2012 & E27 & 2684 & STB E118 \\
\hline 23 Jul 2012 & W140 & 2003 & STA W121 \\
\hline 1 Sep 2014 & E108 & 2216 & STA W161 \\
& & & STB E167 \\
\hline 14 Dec 2014 & W145 & 2222 & STA W172 \\
\hline
\end{tabular}

The time profiles of 13-100 MeV protons for the selected events are compared to that of the 13 Dec 2006 GLE event as a function of time since the associated flare in Figure 2. With the exception of the 4 Jun 2011 event, they all have peak intensities larger than that of 13 Dec 2006. All of the events have fairly rapid rise times, although only the 1 Sep 2014 event (at both STEREOs) exhibits an early peak and decay profile similar to that of 13 Dec 2006; the others have broader peaks and the 14 Dec 2014 event peak occurs later than the rest. We have examined the STEREO shock list maintained by L. Jian (www-ssc.igpp.ucla.edu/ jlan/ STEREO/Level3/ STEREO_Level3_Shock.pdf) and identified the shocks associated with each event. Since the STA shock list currently ends in Aug 2014, we searched the online plasma data plots for evidence of shocks for the 1 Sep 2014 and 14 Dec 2014 STA events; we did not find a convincing candidate for $1 \mathrm{Sep}$ 2014, but identified a possible shock at 05:30 on 15 Dec 2014. Only the $23 \mathrm{Jul} 2012$ event exhibited a peak in the $13-100 \mathrm{MeV}$ proton intensities at the time of the shock passage (see [6] for a more detailed description of this event). This is perhaps not 


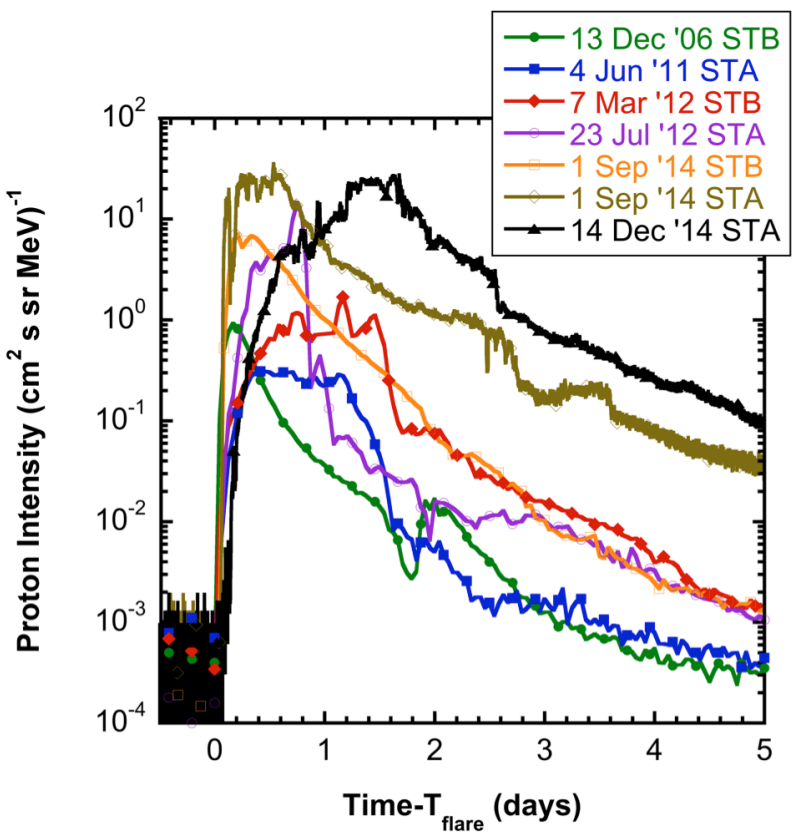

Figure 2. $13-100 \mathrm{MeV}$ proton intensity-time profiles (starting at the time of the associated flare) for the selected events. Intensities from the 13 Dec 2006 event are shown for comparison.

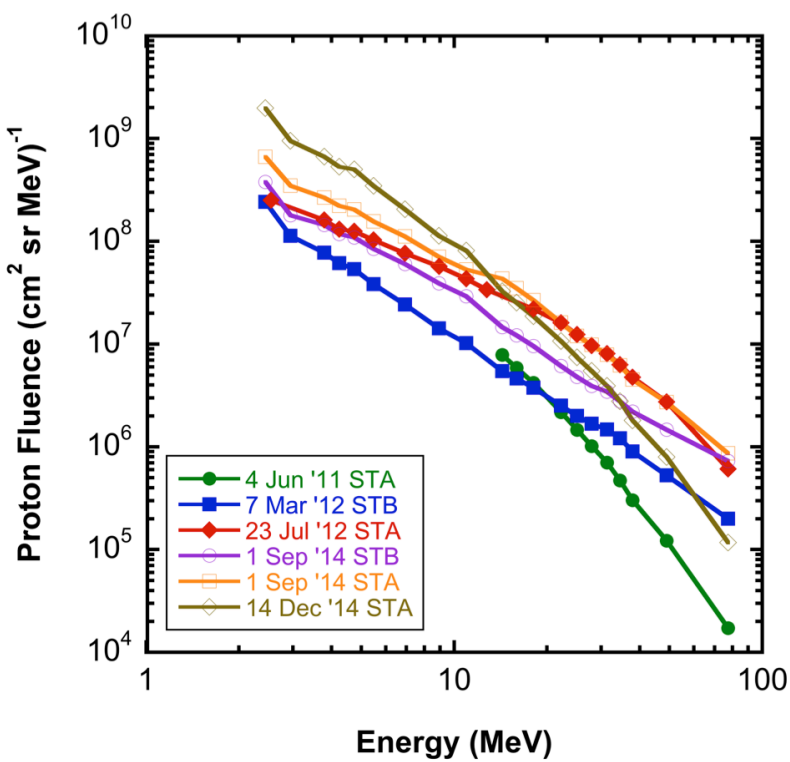

Figure 3. Event-integrated proton fluence spectra for each event. The lower energy portion is missing from 4 Jun 2011 due to a gap in the LET data.

may yield fluences comparable to those of 13 Dec 2006).

As indicated in Figure 4, the three HS events look to have higher fluences at $500 \mathrm{MeV}$ than the two GLE events by factors of $\sim 4$ to 500 (although we cannot rule out additional softening above $100 \mathrm{MeV}$, invalidating our simple extrapolation). This suggests that had the Earth been located where STEREO was during these events, GLEs might have been observed. surprising as although all the events were accompanied by fast CMEs (see Table 1), with the exception of 23 Jul 2012 and 14 Dec 2014, all the events had solar source regions located well west of the spacecraft longitude. SEP events from western sources often do not show strong local shock effects [8].

\subsection{Event Spectra}

We combined LET and HET proton data to create event-integrated fluence spectra for each of the events (note: there are no LET data for the 4 Jun 2011 event due to a data gap); the resulting spectra are given in Figure 3. As can be seen, three of the events exhibit a steepening of the proton fluences above a few tens of MeV: 4 Jun 2011, 23 Jul 2012 and 14 Dec 2014. The other three events, 7 Mar 2012, 1 Sep 2014 STA and STB have harder spectra. We refer to these events/groups as soft spectrum (SS) and hard spectrum (HS) events, respectively. Figure 4 focuses on the $>10 \mathrm{MeV}$ portion of the spectra and compares each group to the last two GLE events (13 Dec 2006 and 17 May 2012). For the HS events we have extrapolated the spectra to $500 \mathrm{MeV}$ for comparison to the $500 \mathrm{MeV}$ fluences of the identified GLE events. It is clear the SS events probably have significantly lower fluences at 500 $\mathrm{MeV}$ (although depending on how the spectrum softens, 23 Jul 2012 

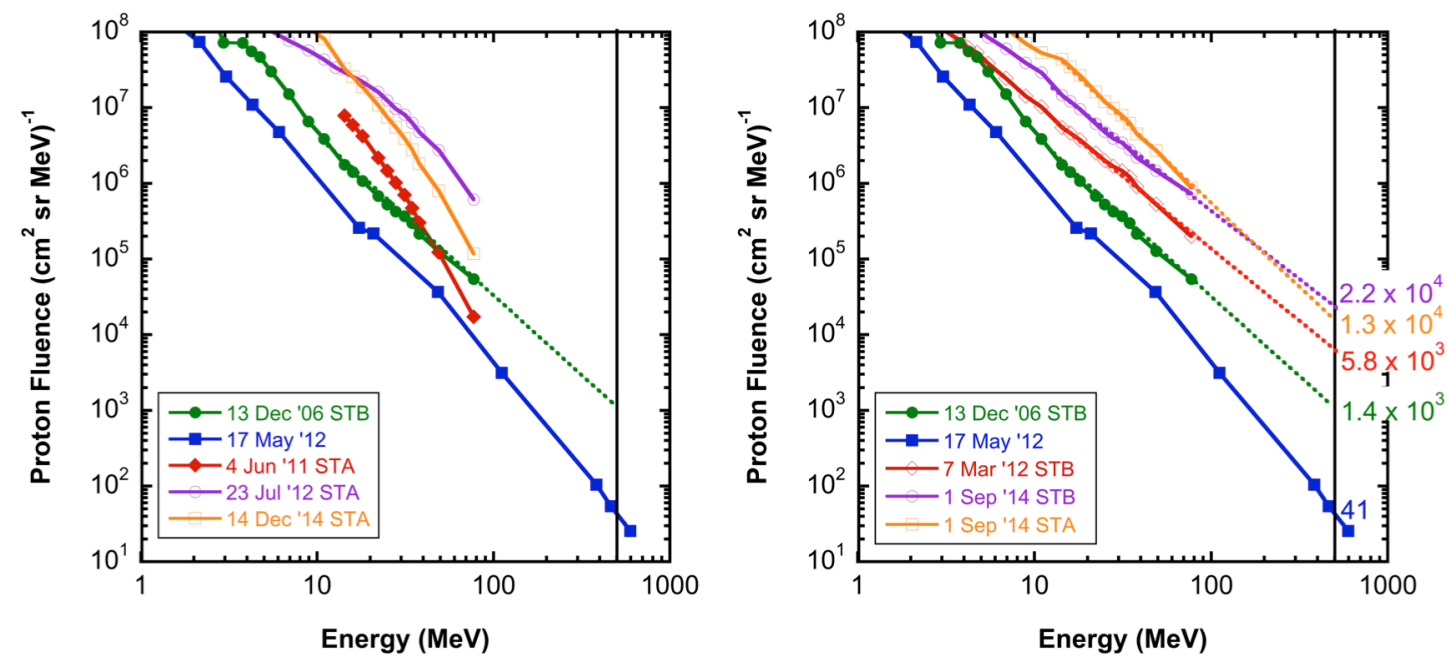

Figure 4. Comparison of the soft spectrum (SS; left) and hard spectrum (HS; right) event spectra to those of the 13 Dec 2006 and 17 May 2012 GLE events. For the HS events, the spectra are extrapolated to $500 \mathrm{MeV}$ (vertical line) to estimate the SEP fluence as compared to those of the two GLE events.

From the extrapolations of the HS event spectra we obtained spectral indices of -2.0, -1.8 and -2.3 for the 7 Mar 2012, 1 Sep 2014 STB and 1 Sep 2014 STA events, respectively. Mewaldt et al. [3] obtained an index of -2.42 (above the break energy) for the 13 Dec 2006 event (Figure 1) and found an average value of -3.18 for their survey of 16 GLE events. Although this average is significantly softer than the values we estimated for the HS events, 9 of the 16 events studied had indices between -2 and -3 . In comparison, only 3 of the 22 large, nonGLE SEP events Mewaldt et al. also examined had spectral indices between -2 and -3 . Thus our HS events are more similar to the GLE group than the non-GLE group.

\subsection{Event Composition}

Mewaldt et al. [3] noticed that GLE events were more likely than large, non-GLE SEP events to have $\mathrm{Ne} / \mathrm{O}$ and $\mathrm{Fe} / \mathrm{O}$ abundance ratios above the average values of 0.152 and 0.134 obtained for 5-12 MeV/n by [9]. We estimated these abundances ratios from the $\mathrm{O}, \mathrm{Ne}$, and $\mathrm{Fe}$ spectra between 10-30 MeV/n using the LET data for our events (Table 2). Only the $7 \mathrm{Mar}$ 2012 event has an enhancement in the $\mathrm{Ne} / \mathrm{O}$ abundance, with the rest of the events exhibiting ratios near or below the Reames values. Further there is no clear difference between the HS and SS events. Mewaldt et al. did find some GLE events with abundances similar to those of our events, including three with both $\mathrm{Ne} / \mathrm{O}$ and $\mathrm{Fe} / \mathrm{O}$ values below those of [9].

Table 2: Composition of Selected Events

\begin{tabular}{|c|c|c|}
\hline Date & Ne/O & $\mathbf{F e} / \mathbf{O}$ \\
\hline 4 Jun 2011 & Data gap & Data gap \\
\hline 7 Mar 2012 & 0.20 & 0.11 \\
\hline 23 Jul 2012 & 0.12 & 0.07 \\
\hline 1 Sep 2014 & 0.15 (STB) & 0.10 (STB) \\
& 0.13 (STA) & 0.07 (STA) \\
\hline 14 Dec 2014 & 0.15 & 0.08 \\
\hline
\end{tabular}

\section{Connection Modeling}

To better understand the observed characteristics of our selected SEP events, we have used models from the Community Coordinated Modeling Center to examine the changing magnetic connections between the observing spacecraft and the CME-driven shock. As discussed in [10] 
and [11], it is clear that accurate simulations of magnetic connection between an observer and a CME-driven shock depends on the quality of the representation of the background solar wind. We have used the combined WSA-ENLIL+Cone model, which is used world-wide for space weather operations and for solar wind and CME simulations, and tuned the model to reproduce the observed solar wind parameters for each event. Included in the simulations were any additional CMEs observed to occur before, during and after the SEP event of interest.

One of the outputs of the WSA-ENLIL+Cone model is the temporal history of the magnetic connection between the shock and the spacecraft. In addition to examining the evolution of the field lines intercepted by the observing spacecraft, we can determine which portions of the ecliptic plane are connected to the shock and experience solar wind speed increases. This is illustrated in the contour plots in Figure 5, where the ambient solar wind solution is colored green, and sectors experiencing speed increases of 20-200, 200-400, and $>400 \mathrm{~km} / \mathrm{s}$ are colored yellow, orange and red, respectively. For each event, the time period shown is when the CME has just emerged and is seen as the small grey crescent near the center.

In all but the 14 Dec 2014 event, the spacecraft is connected to the shock as it enters the simulation at the ENLIL inner boundary at $21.5 \mathrm{R}_{\mathrm{S}}$ (as indicated by the red sector encompassing the observing spacecraft) and there are CMEs prior to the event CME which may pre-condition the heliosphere (although the prior CME for the 4 Jun 2011 event was not directed towards STA). One difference seen in the HS and SS event simulations is the magnetic connection between the observing spacecraft (indicated by the white/black lines) and the CMEs. For all of the HS events (7 Mar 2012 STB, 1 Sep 2014 STA and STB), the spacecraft is connected to both the preceding CME and the event CME. For two of the SS events (4 Jun 2011 and 14 Dec 2014) the spacecraft is only connected to the event CME. Whether the SS event of 23 Jul 2012 follows this trend is not clear. This event is very complicated with multiple preceding CMEs (most of which are not evident in the time period shown in Figure 5). The effects of these CMEs on the magnetic connection between the observing spacecraft and the event CME and the subsequent impact on the observed SEP intensities has been described in detail by [10].

Gopalswamy et al. [12] and Lario and Karelitz [13] have noted that the presence of preceding CMEs can affect the peak intensities of an SEP event. It is possible that multiple magnetic connections and/or the presence of preceding CMEs propagating towards the observing spacecraft, as occurred in the HS events, creates conditions favorable to rapid and efficient acceleration of particles to high energies, e.g., magnetic mirrors or increased turbulence which returns ions to the shock, higher dentsities of suprathermal seed particles, or merging shock complexes. In turn this could increase the likelihood of an SEP event being intense enough and having hard enough spectra to create a GLE. WSA-ENLIL+Cone model simulations of the 13 Dec 2006 and 17 May 2012 GLE events (as well as others) may yield additional insight regarding this. If true, the observation of preceding CMEs could be a valuable contribution to predicting the severity of an SEP event and the occurrence of a GLE.

\section{Conclusions}

We have identified several large SEP events observed by STEREO with 13-100 MeV proton intensities similar to or greater than those observed during the 13 Dec 2006 GLE event. By examining the event-integrated proton spectra above $20 \mathrm{MeV}$ we find that three of the events have hard power law spectra, which when extrapolated to $500 \mathrm{MeV}$, suggest fluence values that 



Figure 5. WSA-ENLIL+Cone model simulations of the five event time periods. The event CMEs are indicated by the grey crescents near the center and preceeding CMEs by the grey crescents outside of 0.5 AU. The yellow, orange and red colors indicate sections of the ecliptic plane that are connected to the shock and have solar wind speed increases of 20-200, 200-400, and $>400 \mathrm{~km} / \mathrm{s}$, respectively. Green shows the ambient solar wind. The position of several spacecraft (e.g., STA, STB) and the Earth are indicated by the colored symbols. The spacecraft of note for each event are, in order, STA, STB, STA, STA + STB, and STA (see Table 1). 
exceed those of the GLE events of 14 Dec 2006 and 17 May 2012 by factors of $\sim 4$ to 500. The spectral indices of these events are similar to those observed for many GLE events of cycle 23, although they do not exhibit enhanced $\mathrm{Fe} / \mathrm{O}$ or $\mathrm{Ne} / \mathrm{O}$ abundance ratios as is sometimes found in GLE events. We conclude that had these events been directed towards Earth is it likely that they would have created GLEs. Our remaining events, while large, had spectra that steepened significantly above a few tens of $\mathrm{MeV}$ such that their fluences at $500 \mathrm{MeV}$ were likely much lower than those of the GLE events.

We used the WSA-ENLIL+Cone model to investigate the evolution of the inner heliosphere during these events, specifically the magnetic connections between the observers and the CME-related shocks. We find that during the three events with hard spectra the observing spacecraft had magnetic connection to the event CME very early while also having connection to a preceding CME. It is possible that this configuration led to an increased efficiency in accelerating particles to high energies and may be something to consider when predicting the occurence of extreme SEP events and/or GLEs.

\section{References}

[1] Lopate, C., Fifty years of ground level solar particle event observations, in Solar Eruptions and Energetic Particles, ed. by N. Gopalswamy, R.A. Mewaldt, J. Torsti, AGU Mono. Ser. (2006) p. 283

[2] Gopalswamy, N. et al., The first ground level enhancement event of solar cycle 24: Direct observation of shock formation and particle release heights, ApJ 765 (2013) L30

[3] Mewaldt, R.A. et al., Energy spectra, composition and other properties of ground-level events during solar cycle 23, SSRv 171 (2012) 97

[4] Band, D. et al., BATSE observations of gamma-ray burst spectra. I - Spectral diversity, ApJ 413 (1993) 281

[5] Gopalswamy, N. et al., Properties of ground level enhancement events and the associated solar eruptions during solar cycle 23, SSRv 171 (2012) 23

[6] Russell, C.T. et al., The very unusual interplanetary coronal mass ejection of 2012 July 23; A blast wave mediated by solar energetic particles, ApJ 770 (2013) 10.1088/0004-637X/770/1/38

[7] Gopalswamy, N. et al., The 2012 July 23 backside eruption: An extreme energetic particle event?, ApJ 833 (2016) 10.3847/1538-4357/833/2/216

[8] Cane, H.V. et al., The role of interplanetary shocks in the longitude distribution of solar energetic particles, JGR 93 (1988) 9555

[9] Reames, D.V., Coronal abundances determined from energetic particles, ASR 15 (1995) 41

[10] Bain, H.M. et al., Shock connectivity in the August 2010 and July 2012 solar energetic particle events inferred from observations and ENLIL modeling, ApJ 825 (2016) 10.3847/0004$637 \mathrm{X} / 825 / 1 / 1$

[11] Mays, M.L. et al., Ensemble modeling of CMEs using the WSA-ENLIL+Cone model, SoPh 290 (2015) 1775

[12] Lario, D. and A. Karelitz, Influence of interplanetary coronal mass ejections on the peak intensity of solar energetic particle events, JGR (2014) 10.1002/2014JA019771

[13] Gopalswamy, N. et al., Intensity variation of large solar energetic particle events associated with coronal mass ejections, JGR (2004) 10.1029/2004JA010602 